\title{
On a discrete variant of Bernstein's polynomial inequality
}

\author{
Dimiter Dryanov Richard Fournier
}

\section{CRM-3178}

January 2005 

Abstract

It is known that for any polynomial $p$ of degree less or equal to $n$,

$$
\max _{|z| \leq 1}\left|p^{\prime}(z)\right| \leq n \max _{0 \leq j \leq 2 n-1}\left|p\left(e^{i j \pi / n}\right)\right|
$$

In this note we establish cases of equality and also improve the above inequality.

AMS 1991 Subject Classification: 30C10, 41A17

Key Words and Phrases: complex polynomials, Bernstein inequality. 



\section{Introduction}

Let $\mathcal{P}_{n}$ be the class of polynomials $p(z):=\sum_{k=0}^{n} a_{k}(p) z^{k}$ of degree at most $n$ with complex coefficients. We write, together with $\mathbb{D}:=\{z|| z \mid<1\}$,

$$
|p|_{\mathbb{D}}:=\max _{|z|=1}|p(z)|
$$

and

$$
|p|_{[-1,1]}:=\max _{-1 \leq x \leq 1}|p(x)|
$$

According to the famous inequalities of Bernstein and Markov we have

$$
\left|p^{\prime}\right|_{\mathbb{D}} \leq n|p|_{\mathbb{D}}
$$

and

$$
\left|p^{\prime}\right|_{[-1,1]} \leq n^{2}|p|_{[-1,1]} .
$$

We refer the reader to the book by Rahman and Schmeisser [4] for an exhaustive list of references concerning (1) and (2).

We shall be concerned in this note by the following discrete refinements of (1) and (2):

$$
\left|p^{\prime}\right|_{\mathbb{D}} \leq n \max _{1 \leq j \leq 2 n}\left|p\left(e^{i j \pi / n}\right)\right|
$$

and

$$
\left|p^{\prime}\right|_{[-1,1]} \leq n^{2} \max _{0 \leq j \leq n}|p(\cos (j \pi / n))| .
$$

The inequality (4) is due to Duffin and Schaeffer [2] and it is known that equality holds in (4) only for multiples of the $n$th Chebyshev polynomial $T_{n} \in \mathcal{P}_{n}$ which is defined by

$$
T_{n}(x):=\cos (n \arccos (x)), \quad x \in[-1,1] .
$$

The more recent inequality (3) is due to Frappier, Rahman and Ruscheweyh [3]. However it does not seem to be known for which polynomials equality holds in (3). The main result of this note settles this question and contains an improvement of (3). We shall prove

Theorem 1 Let $n \geq 1$ and $p(z):=\sum_{k=0}^{2 n} a_{k}(p) z^{k} \in \mathcal{P}_{2 n}$. Then for any real $\theta$,

$$
\left|e^{i \theta} p^{\prime}\left(e^{i \theta}\right)-2 \sum_{k=1}^{n} k a_{n+k}(p) e^{i(n+k) \theta}\right| \leq n \max _{1 \leq j \leq 2 n}\left|p\left(e^{i j \pi / n}\right)\right| .
$$

Further, the equality

$$
\left|p^{\prime}\right|_{\mathbb{D}}=n \max _{1 \leq j \leq 2 n}\left|p\left(e^{i j \pi / n}\right)\right|
$$

holds for some $p \in \mathcal{P}_{n}$ if and only if

$$
p(z) \equiv K z^{n}, \quad \text { for some complex number } K .
$$

\section{Proof of Theorem 1}

We shall need two auxiliary results. Let $\mathcal{T}_{n}$ denote the class of trigonometric polynomials

$$
t(\theta):=\sum_{k=-n}^{n} a_{k} e^{i k \theta}
$$

with complex coefficients. Then we have

Lemma 1 For any $t \in \mathcal{T}_{4 n-1}$ and $\gamma \in \mathbb{R}$,

$$
\frac{1}{2 \pi} \int_{-\pi}^{\pi} t(\theta) d \theta=\frac{1}{4 n} \sum_{j=0}^{4 n-1} t\left(\left(\frac{j \pi}{2}-\gamma\right) / n\right) .
$$


Lemma 1 is a well-known quadrature formula (see for example [1] as a ready reference). Simple computations show that the quadrature is valid for $t(\theta):=e^{i k \theta}, 0 \leq k \leq 4 n-1$. We shall also need

Lemma 2 For any $p \in \mathcal{P}_{n}$ and $\gamma, \varphi \in \mathcal{R}$,

$$
e^{i(\gamma+\varphi)} p^{\prime}\left(e^{i \varphi}\right)=\frac{1}{\pi} \int_{-\pi}^{\pi} \cos (n v+\gamma) \frac{1-\cos (n v)}{1-\cos (v)} p\left(e^{i(\varphi-v)}\right) d v .
$$

This last lemma is, to the best of our knowledge, new and again simple computations show that it is valid for $p(z):=z^{k}, 0 \leq k \leq n$.

We first prove our theorem for $p \in \mathcal{P}_{n}$. Indeed, we have for $\gamma, \varphi \in \mathbb{R}$,

$$
e^{i(\gamma+\varphi)} p^{\prime}\left(e^{i \varphi}\right)=\frac{1}{2 n} \sum_{k=0}^{2 n-1}(-1)^{k} \ell_{k} p\left(e^{i(\varphi-k \pi / n+\gamma / n)}\right)
$$

with $\ell_{k}=\ell_{k}(\gamma)=\frac{1-\cos (k \pi-\gamma)}{1-\cos \left(\frac{k \pi}{n}-\frac{\gamma}{n}\right)} \geq 0$ and $\frac{1}{2 n} \sum_{k=0}^{2 n-1} \ell_{k}=n$. This can been seen as follows: by Lemma 2,

$$
e^{i(\gamma+\varphi)} p^{\prime}\left(e^{i \varphi}\right)=\frac{1}{\pi} \int_{-\pi}^{\pi} \cos (n v+\gamma)\left[n+\sum_{j=1}^{n-1}(n-j)\left(e^{i j v}+e^{-i j v}\right)\right] p\left(e^{i(\varphi-v)}\right) d v
$$

and we remark that the integrand in (6) is a trigonometric polynomial $t\left(e^{i v}\right)$ of degree at most $3 n-1 \leq 4 n$. We now use Lemma 1 and get

$$
\begin{aligned}
e^{i(\gamma+\varphi)} p^{\prime}\left(e^{i \varphi}\right) & =\frac{1}{2 n} \sum_{j=0}^{4 n-1} \cos \left(\frac{j \pi}{2}\right) \frac{1-\cos \left(\frac{j \pi}{2}-\gamma\right)}{1-\cos \left(\frac{j \pi}{2 n}-\frac{\gamma}{n}\right)} p\left(e^{i(\varphi-j \pi / 2 n+\gamma / n)}\right) \\
& =\frac{1}{2 n} \sum_{k=0}^{2 n-1}(-1)^{k} \frac{1-\cos (k \pi-\gamma)}{1-\cos \left(\frac{k \pi}{n}-\frac{\gamma}{n}\right)} p\left(e^{i(\varphi-k \pi / n+\gamma / n)}\right)
\end{aligned}
$$

The substitution $p(z):=z^{n}$ in (7) yields

$$
\frac{1}{2 n} \sum_{k=0}^{2 n-1} \ell_{k}=n
$$

We remark that (5) is a special case of an identity proved in [3, Lemma $\left.4^{\prime}\right]$, although the coefficients $\ell_{k}$ where not explicitly determined there. It is now seen that (3) is a simple consequence of (7): let $p \in \mathcal{P}_{n}, \varphi \in \mathbb{R}$ and $\gamma=-n \varphi$. Then

$$
\begin{aligned}
\left|p^{\prime}\left(e^{i \varphi}\right)\right| & =\left|e^{i(\gamma+\varphi)} p^{\prime}\left(e^{i \varphi}\right)\right| \\
& =\left|\frac{1}{2 n} \sum_{k=0}^{2 n-1} \ell_{k}(-1)^{k} p\left(e^{-i k \pi / n}\right)\right| \\
& \leq \frac{1}{2 n} \sum_{k=0}^{2 n-1} \ell_{k}\left|p\left(e^{-i k \pi / n}\right)\right| \\
& \leq\left(\frac{1}{2 n} \sum_{k=0}^{2 n-1} \ell_{k}\right) \max _{0 \leq j \leq 2 n-1}\left|p\left(e^{i j \pi / n}\right)\right| \\
& =n \max _{0 \leq j \leq 2 n-1}\left|p\left(e^{i j \pi / n}\right)\right| .
\end{aligned}
$$

Let now $p \in \mathcal{P}_{n}$ such that equality holds in (3), i.e., $\varphi \in \mathbb{R}$ and

$$
\left|p^{\prime}\right|_{\mathbb{D}}=\left|p^{\prime}\left(e^{i \varphi}\right)\right|=n \max _{0 \leq j \leq 2 n-1}\left|p\left(e^{i j \pi / n}\right)\right| .
$$

We assume first that $|\cos (n \varphi)|<1$. Then

$$
\ell_{k}=\frac{1-(-1)^{k} \cos (n \varphi)}{1-\cos (k \pi / n-\varphi)}>0, \quad 0 \leq k \leq 2 n-1
$$


and equality must hold everywhere in (8). Because of the equality case in the triangle inequality, we obtain that

$$
(-1)^{k} p\left(e^{-i k \pi / n}\right)=M e^{i \psi}, \quad 0 \leq k \leq 2 n-1
$$

where $\psi$ is a fixed real number and $M:=\max _{1 \leq j \leq 2 n}\left|p\left(e^{i j \pi / n}\right)\right|$. This amounts to the fact that the polynomial $q \in \mathcal{P}_{n}$ defined by

$$
q(z)=p(z)-M e^{i \psi} z^{n}
$$

has a zero at each $(2 n)$ th-root of unity and therefore $q \equiv 0$, i.e., $p(z) \equiv M e^{i \psi} z^{n}$. We now have to discuss the case where $|\cos (n \varphi)|=1$. We may without any loss of generality assume that $\varphi=0$ because the set of $(2 n)$ th-roots of unity is closed under multiplication by one of its members. We obtain as in (8)

$$
\begin{aligned}
n M=\left|p^{\prime}(1)\right| & =\left|\frac{1}{2 n} \sum_{k=0}^{2 n-1}(-1)^{k} \frac{1-\cos (k \pi)}{1-\cos (k \pi / n)} p\left(e^{-i k \pi / n}\right)\right| \\
& =\left|\frac{n}{2} p(1)+\frac{1}{2 n} \sum_{\substack{k=1 \\
k \text { odd }}}^{2 n-1} \frac{2}{1-\cos (k \pi / n)}\left(-p\left(e^{-i k \pi / n}\right)\right)\right| \\
& \leq\left(\frac{n}{2}+\frac{1}{2 n} \sum_{\substack{k=1 \\
k \text { odd }}}^{2 n-1} \frac{2}{1-\cos (k \pi / n)}\right) M \\
& =n M, \quad
\end{aligned}
$$

i.e., equality holds everywhere in (9) and since none of the numbers $\frac{2}{1-\cos (k \pi / n)}(1 \leq k \leq 2 n-1, k$ odd $)$ vanishes, there exists a real number $\psi$ such that

$$
q(z)=p(z)-M e^{i \psi} z^{n} \in \mathcal{P}_{n}
$$

has a zero at each of the $n+1$ distinct points $e^{-i k \pi / n},(0 \leq k \leq 2 n-1, k=0$ or $k$ is odd $)$. Just as above $p(z) \equiv M e^{i \psi} z^{n}$.

We now have to verify our claim concerning $\mathcal{P}_{2 n}$. Let $P(z)=\sum_{k=0}^{2 n} a_{k}(P) z^{k} \in \mathcal{P}_{2 n}$. Applying Lemma 2 to $p(z):=P(z)-\sum_{k=n+1}^{2 n} a_{k}(P) z^{k} \in \mathcal{P}_{n}$, we obtain after some simple computations

$$
e^{i \theta} P^{\prime}\left(e^{i \theta}\right)-2 \sum_{k=1}^{n} k a_{n+k}(P) e^{i(n+k) \theta}
$$

$$
=\frac{1}{\pi} \int_{-\pi}^{\pi} \cos (n v+\gamma) \frac{1-\cos (n v)}{1-\cos (v)} P\left(e^{i(\theta-v)}\right) d v .
$$

The integrand in (10) is a trigonometric polynomial of degree at most $4 n-1$. Upon an application of Lemma 1 we obtain (with $\gamma=-n \theta$ )

$$
e^{i \theta} P^{\prime}\left(e^{i \theta}\right)-2 \sum_{k=1}^{n} k a_{n+k}(P) e^{i(n+k) \theta}=\frac{1}{2 n} \sum_{k=0}^{2 n-1} \ell_{k}(-1)^{k} P\left(e^{-i k \pi / n}\right) .
$$

The result follows just as above. It is however of interest to notice that the equality

$$
\left|e^{i \theta} P^{\prime}\left(e^{i \theta}\right)-2 \sum_{k=1}^{n} k a_{n+k}(P) e^{i(n+k) \theta}\right|=n \max _{0 \leq j \leq 2 n-1}\left|P\left(e^{i j \pi / n}\right)\right|
$$

holds for all real numbers $\theta$ and all polynomials with complex coefficient $a, b$ of the type

$$
P(z)=a z^{2 n}+b z^{n}-a, \quad z=e^{i \theta} .
$$

\section{References}

[1] D.P. Dryanov. Quadrature formulae with free nodes for periodic functions. Numer. Math., 67:441-464, 1994. 
[2] R.J. Duffin and A.C. Schaeffer. A refinement of an inequality of the brothers Markoff. Trans. Amer. Math. Soc., 50:517-528, 1941.

[3] C. Frappier, Q.I. Rahman, and St. Ruscheweyh. New inequalities for polynomials. Amer. Math. Soc. 288:69-99, 1985.

[4] Q.I. Rahman and G. Schmeisser. Analytic Theory of Polynomials. Oxford University Press, Oxford, 2002.

\section{Dryanov}

Department of Mathematics and Statistics

Concordia University

1455 de Maisonneuve West

Montréal H3G 1M8, Canada

ddryanov@alcor.concordia.ca
R. Fournier

Département de Mathématiques et de Statistique

Université de Montréal

C.P. 6128 , succ. Centre-ville

Montréal H3C 3J7, Canada

fournier@dms.umontreal.ca 